\title{
Defining niche interactions to target chronic myeloid leukemia stem cells
}

\section{Rebecca Mitchell and Mhairi Copland}

Paul O'Gorman Leukaemia Research Centre, Institute of Cancer Sciences, University of Glasgow, Glasgow, UK

E-mail: MHAIRI COPLAND - mhairi.copland@glasgow.ac.uk

doi:10.3324/haematol.2019.234898

I n this issue of Haematologica, Godavarthy et al. ${ }^{1}$ describe how the expression of CD44 on the surface of leukemic stem cells (LSC) and E-selectin on bone marrow (BM) endothelium are essential for the engraftment of LSC within the BM niche. They show how this interaction can provide the LSC with protection from imatinib treatment. Importantly, disruption of this axis using an E-selectin inhibitor in combination with imatinib prevents LSC binding to the endothelium and demonstrates superior eradication of LSC in chronic myeloid leukemia (CML) compared with imatinib alone.

CML has been well characterized for a number of years, since the discovery of the Philadelphia chromosome and associated aberrant BCR-ABL signaling. This led to the revolutionary development of BCR-ABL-specific tyrosine kinase inhibitors such as imatinib. Tyrosine kinase inhibitors are highly effective at reducing the leukemic burden and disease progression, inducing remission and prolonging survival of patients with chronic phase CML. ${ }^{2}$ Although very good control over the disease is gained in most chronic phase CML patients with tyrosine kinase inhibitor treatment, eradication of the LSC population, which sustains and repopulates the disease in patients, remains elusive.

It has been established that the BM niche provides a sanctuary for LSC in which to thrive and avoid pharmacological interventions. There have been many studies showing how the niche adapts and is exploited during leukemic transformation. ${ }^{4 \cdot 6}$ By gaining, a better understanding of the interactions between LSC and their microenvironment, it may be possible to distinguish factors that favor survival of the leukemic cells and identify targets for improved drug therapy. One such strategy is to inhibit the homing and engraftment of LSC within the BM niche, without affecting normal hematopoietic stem cells (HSC).

The BM microenvironment is complex, due to the array of different cell types which reside there, including osteolineage cells, mesenchymal stem cells, endothelial cells, neurons and hematopoietic cells. ${ }^{8}$ In order to control and maintain homeostasis of the healthy BM niche, a multitude of cytokines and chemokines help determine HSC fate. ${ }^{8}$ In normal hematopoiesis homing and engraftment of HSC within the BM is a highly coordinated multistep process that requires activation of different adhesion receptors to maintain tight regulation. Selectins and integrins are very important within this process. ${ }^{9,10}$ Expression of integrins allows HSC to bind to vascular cell adhesion molecule- 1 on BM endothelium and fibronectin on the extracellular matrix. Integrins, such as VLA4, also interact with E- and Pselectins, which are found constitutively expressed on the BM endothelium, and this interaction mediates HSC rolling and homing. ${ }^{10}$ Another important factor within this process is CXCL12; this chemkine functions as an HSC chemoattractant through its receptor CXCR4, which through crosstalk with $\beta_{1}$ and $\beta_{2}$ integrins, mediates HSC homing and is required for stable engraftment. ${ }^{9} \mathrm{CD} 44$ has also been linked to HSC homing; however, HSC that do not express CD44 can still home and engraft normally. ${ }^{11}$

In studies comparing the homing and engraftment of CML LSC to normal HSC, it has been shown that patients with CML have several adhesion abnormalities. ${ }^{12,13}$ LSC have defective $\beta_{1}$ integrin function, despite normal expression of VLA4 and VLA5, which decreases LSC adhesion to BM stroma. ${ }^{13}$ It has also been shown that primary CML progenitor cells have reduced CXCR4 expression and impaired chemotaxis towards CXCL12, as well as reduced CXCL12-mediated integrin adhesion. ${ }^{12}$ Despite these functional defects, LSC can still home and engraft within the $\mathrm{BM}$ and therefore must use alternative mechanisms.

Godavarthy et al. ${ }^{1}$ demonstrate a novel reciprocal link between the external cues from the BM microenvironment and BCR-ABL-specific LSC-intrinsic pathways. They show how this leads to modulation of the expression of LSC adhesion molecules and alters interactions with the vascular niche. The authors confirm the interaction of $\mathrm{BCR}-\mathrm{ABL}^{+}$ cells and the vascular endothelium by using very sophisticated in vivo microscopy of the calvarium of mice injected with human CML leukocytes. Using time-lapse imaging, they found that the contact time of the leukemic cells to the $\mathrm{BM}$ endothelium was reduced in mice treated with an Eselectin inhibitor (GMI-1271).

The authors replicated these findings in a transgenic model, showing that $\mathrm{BCR}-\mathrm{ABL}^{+}$cells were situated significantly further away from the endothelium if treated with GMI-1271 and imatinib. Furthermore, after treatment with GMI-1271 and imatinib, mice had improved survival, and demonstrated reduced numbers of CML-initiating clones, impaired short-term homing to the spleen and the BM, reduced leukocyte counts, $\mathrm{BCR}-\mathrm{ABL}^{+}$myeloid cell counts and spleen size. Further studies showed that inhibition of Eselectin led to non-adhesion and an increase of Scl/Tall expression in $\mathrm{BCR}-\mathrm{ABL}^{+}$leukemia-initiating cells (LIC) in vitro. Additionally, Scl/Tal1 negatively regulated the expression of CD44 on LIC, and overexpression of Scl/Tall on LIC led to prolongation of survival in a murine model of CML, similar to the improved survival with CD44-deficient CML-initiating cells previously demonstrated by Krause et al. $^{14}$

In vitro experiments demonstrated that CD44 was highly expressed in BCR-ABL ${ }^{+}$cells compared to $\mathrm{BCR}-\mathrm{ABL}^{-}$cells, and that when $\mathrm{BCR}-\mathrm{ABL}^{+}$cells were treated with GMI1271 alone or in combination with imatinib there was an increase in cells in G2-S-M phase and a decrease in the G0 phase of the cell cycle. This coincided with an increase in cell cycle promoter CDK6 and decreased expression of cell cycle inhibitor $\mathrm{p} 16$. Furthermore, BCR-ABL1 phosphorylated SCL/TAL1 via the AKT signaling pathway. SCL/TAL1 regulated the activity of the CD44 regulatory element by 


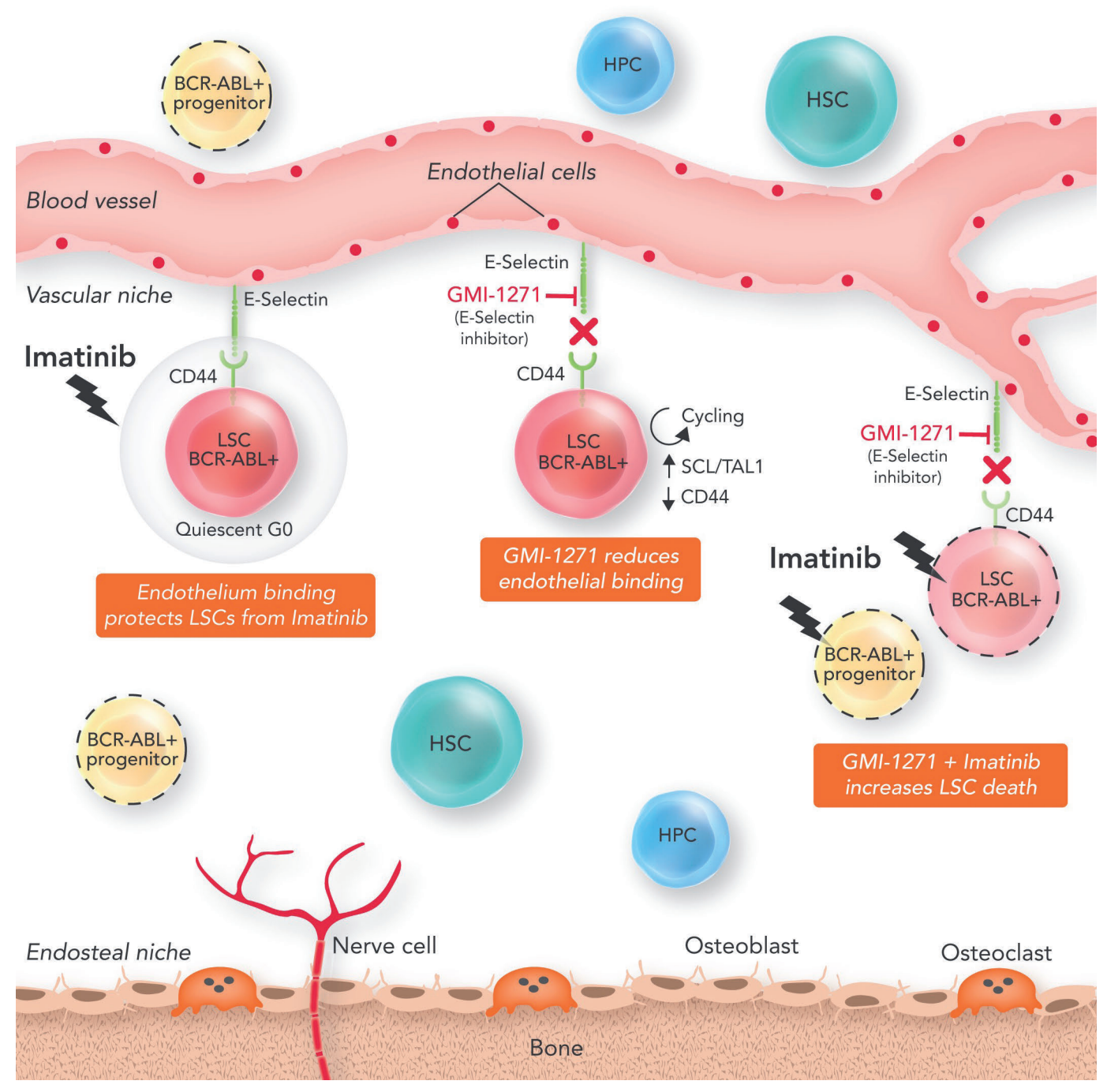

Figure 1. This schematic demonstrates the effects of E-selectin inhibition within the bone marrow microenvironment and the impact upon chronic myeloid leukemia stem cells and mechanistically how this is controlled by the SCL/TAL1 - CD44 axis. HPC: hematopoietic progenitor cell; HSC; hematopoietic stem cell; LSC: leukemic stem cell.

acting as a transcriptional repressor leading to decreased expression of CD44, decreased adhesion to the vascular niche and an increase in cycling LSC.

Interestingly, higher expression of CD44 was demonstrated in BCR-ABL cells specifically harboring the T315I mutation, which correlated with increased binding to Eselectin, with a larger amount of adherent cells in G0. The authors suggest that the increased expression of CD44 and increased binding to E-selectin may contribute to LSC dormancy and resistance to tyrosine kinase inhibitors.

Finally, relevance to human CML was established as leukocytes from patients with CML had higher transcriptional expression of SCL/TAL1 and lower CD44 expression compared to those from healthy individuals. Analyses of published datasets suggest a trend that expression of SCL/TAL1 and CD44 may correlate with disease stage and survival in CML patients; however, larger cohorts and further experimental data are required to confirm this.

The important experiments presented by Godavarthy et al. establish the mechanism of increased expression of
CD44 on BCR-ABL1 ${ }^{+}$cells. They further showed that dislocation of $\mathrm{BCR}-\mathrm{ABL} 1^{+}$cells from the niche, via inhibition of E-selectin binding, increased $\mathrm{BCR}-\mathrm{ABL}^{+}$cell cycle progression and increased responsiveness to imatinib therapy. ${ }^{1}$

Inhibition of E-selectin has been shown to have therapeutic utility in other cancer types, such as acute myeloid leukemia and solid tumors in which it is thought to have a role in metastasis. ${ }^{15}$ In acute myeloid leukemia, the leukemic blast cells bind to E-selectin on the endothelium and this activates leukemic pathways that contribute to chemotherapy resistance. ${ }^{16}$ Currently, GMI-1271 is in a phase I/II clinical trial to treat acute myeloid leukemia in combination with chemotherapy to disrupt leukemia survival pathways and sensitize the leukemic cells to chemotherapy (ClinicalTrials.gov Identifier: NCT02306291)

E-selectin has also been implicated in the development of metastasis to the lungs from primary solid tumors, such as breast $^{17}$ and colon $^{18}$ cancer. It is hypothesized that, during the premetastatic stage, the primary tumors secrete soluble factors, which induce an inflammatory response in the 
Editorials

blood vessels and activate E-selectin on the endothelium, allowing engraftment of immune progenitor cells. This intaal binding of E-selectin to its ligand confers firm adhesion, and triggers signaling that leads to permabilization of the endothelium through the dissociation of VE-cadherin/ $\beta$ catenin. In an attempt to counteract metastasis, the Eselection inhibitor GMI-1271 is currently being tested in poreclinical models and is showing high efficacy. ${ }^{17}$ As well as being a therapeutic target, E-selectin is also being screened as a potential biomarker for disease progression and measbasis. ${ }^{19}$

The BM microenvironment is a developing research focus which is showing great importance in disease pathophysiology. Advances in technology, such as the time-lapse intra-vital imaging used in the study by Godavarthy et al., ${ }^{1}$ are enabling a greater understanding of the interactions and mechanisms involved in cellular microenvironments This type of pioneering microscopy allows us to see interactions between leukemic cells and the niche, and is providing powerful data, as exemplified by this study as well as many others. ${ }^{13}$ Enriching these data, single-cell RNA-sequencing allows for the analysis of the different cell types within the niche, evaluation of their transcriptional regulation and a view of how they may contribute to disease progression, providing important information which may have been masked using bulk sequencing approaches. ${ }^{20}$ Through these studies, we continue to build upon our knowledge of the pathophysiology of CML and come ever closer to finding a way of eradicating quiescent LSC.

References

1. Godavarthy PS, Kumar R, Herkt SC, et al. The vascular bone marrow niche influences outcome in chronic myeloid leukemia via the Eselection - SCL/TAL1 - CD44 axis . Haematological. 2019;05(1):136-147.

2. Hochhaus A, Larson RA, Guilhot F, et al. Long-term outcomes of inatinib treatment for chronic myeloid leukemia. N Angl J Med. 2017;376(10):917-927.

3. Graham SM, Jørgensen HG, Allan E, et al. Primitive, quiescent, Philadelphia-positive stem cells from patients with chronic myeloid leukemia are insensitive to STI571 in vito. Blood. 2002;99(1):319-325.
4. Ishikawa F, Yoshida S, Saito Y, et al. Chemotherapy-resistant human AML stem cells home to and engraft within the bone-marrow endosteal region. Nat Biotechnol. 2007;25(11):1315-1321.

5. Schemers K, Pietras EM, Reynaud D, et al. Myeloproliferative neoplasia remodels the endosteal bone marrow niche into a self-reinforcing leukemic niche. Cell Stem Cell. 2013;13(3):285-299.

6. Werner RS, Amabile G, Bararia D, et al. Treatment of chronic myelogenous leukemia by blocking cytokine alterations found in normal stem and progenitor cells. Cancer Cell. 2015;27(5):671-681.

7. Krause DS, Lawarides K, Lewis JB, won Adrian UH, Van Etten RA. Selecting and their ligands are required for homing and engraftment of BCR-ABL1+ leukemic stem cells in the bone marrow niche. Blood. 2014;123(9):1361-1371.

8. Morrison SJ, Sadden DT. The bone marrow niche for haematopoietic stem cells. Nature. 2014;505(7483):327-334.

9. Piled A, Kollet O, Ponomaryov T, et al. The chemokine SDF-1 activates the integrins LFA-1, VLA-4, and VLA-5 on immature human CD34+ cells: role in transendothelial/stromal migration and engraftment of NOD/SCID mice. Blood. 2000;95(11):3289-3296.

10. Frenette PS, Subbarao S, Marzo IB, won Adrian UH, Wagner DD. Endothelial selections and vascular cell adhesion molecule-1 promote hematopoietic progenitor homing to bone marrow. Proc Natl Acad Sci U S A. 1998;95(24):14423-14428.

11. Oostendorp RAJ, Ghaffari S, Eaves CJ. Kinetics of in vino homing and recruitment into cycle of hematopoietic cells are organ-specific but CD44-independent. Bone Marrow Transplant. 2000;26(5):559-566.

12. Chang B, Ho YW, Huang $Q$, et al. Altered microenvironmental regulartron of leukemic and normal stem cells in chronic myelogenous leukemia. Cancer Cell. 2012;21(4):577-592.

13. Bhatia R, Verfaillie CM. The effect of interferon- $\alpha$ on beta-1 integrin mediated adhesion and growth regulation in chronic myelogenous leukemia. Leek Lymphoma. 1998;28(3-4):241-254.

14. Krause DS, Lazarides K, won Adrian UH, Van Etten RA. Requirement for CD44 in homing and engraftment of BCR-ABL-expressing leukemic stem cells. Nat Med. 2006;12(10):1175-1180.

15. Erbani J, Barbier V, Lowe J, Thy J, Levesque JP, Winkles I. Vascular niche E-selectin promotes acute myeloid leukemia resistance to chemotheraby via its receptors CD44 and CD162. Exp. Hematol. 2018;64:S63.

16. Chen S, Hag SU, Pawlus M, et al. Adhesion of acute myeloid leukemia blasts to E-selectin in the vascular niche enhances Their Survival By Mechanisms Such As Wit Activation. Blood. 2013;122(21):61.

17. Kans SA, Blache CA, Bajana S, et al. The effect of soluble E-selectin on tumor progression and metastasis. BMC Cancer. 2016;16:331.

18. Köhler S, Ullrich S, Richter U, Schumacher U. E-/P-selectins and colon carcinoma metastasis: first in vive evidence for their crucial role in a clinically relevant model of spontaneous metastasis formation in the lung. Br J Cancer. 2010;102(3):602-609.

19. Pref S, Salami O, Al-Tonbary Y, Fouda M, Menessy A, El-Sherbiny M. $\mathrm{L}$ and $\mathrm{E}$ selections in acute myeloid leukemia: expression, clinical ralevance and relation to patient outcome. Hematology. 2002;7(2):83-87.

20. Tikhonova AN, Dolgalev I, Mu H, et al. The bone marrow microenvironment at single-cell resolution. Nature. 2019;569(7755):222-228.

4

haematological | 2020; 105(1) 